\title{
SÍNDROME ADYACENTE
}

\author{
SÍNDROME ADJACENTE
}

ADJACENT SEGMENT DISEASE

Gustavo Martin' ${ }^{1}$ Oliver Gille ${ }^{2}$, Jean Marc Vital ${ }^{2}$

\begin{abstract}
RESUMEN
Objetivo: Estudiamos la incidencia de SDA quirúrgico de localización lumbar y los factores relacionados con el mismo. Método: Estudio retrospectivo de corte transversal. Resultados: Encontramos 50 casos operados por SDA en 1662 cirugías realizadas, 530 en columna lumbar; incidencia 9,4\%. Edad promedio 62 años. Síntoma principal: claudicación radicular (80\%). Las Rx previas a la primera cirugía

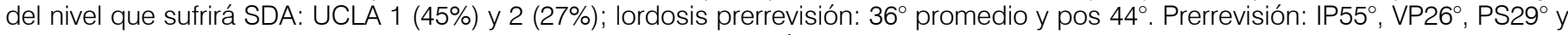
posoperatoria IP56 ${ }^{\circ}, \mathrm{VP} 22^{\circ}$ y PS34 ${ }^{\circ}$. Desequilibrio sagital prerrevisión $60 \%$ y posoperatorio $27 \%$, equilibrio coronal recuperado en los 2 casos. IRM previa a la primera intervención mostró 83\% con Pfirmann IV. Tipos de SDA: estenosis lumbar monosegmentaria agravada por antelistesis en 20 y por retrolistesis en 14; estenosis multisegmentaria 4, hernia discal 4, fractura por aplastamiento 4, estenosis sin listesis 4. Período entre las 2 cirugías: 6 años promedio. Conclusión: Una incidencia de 9,4\% de reintervenciones a causa de un Síndrome Adyacente, hace de esta patología un importante problema a largo plazo, lo que obligaría al cirujano a tomar las medidas que sean posibles para evitarlo. Con los resultados de este estudio retrospectivo solo podríamos decir que tendrían más posibilidades de desarrollar un SDA los segmentos con Pfirmann IV en la IRM y que se debería tener en cuenta las relaciones VP (20\%) y PS (80\%) pre y pos operatorias para devolver las condiciones mecánicas ideales al raquis. Se necesitarían estudios clínicos prospectivos para tener conclusiones más firmes.
\end{abstract}

Descriptores: Síndrome; Enfermedades de la columna vertebral, Cirugía; Radiografía.

\section{RESUMO}

Objetivo: Estudamos a incidência de SA de localização lombar tratada por cirurgia e fatores associados. Método: Estudo transversal retrospectivo. Resultados: Foram encontrados 50 casos de SA em 1.662 casos operados, 530 na coluna lombar, incidência de 9,4\%. A média de idade foi 62 anos. Principais sintomas: claudicação radicular (80\%). Radiografias antes do primeiro nível que sofrerá SA: UCLA 1 (45\%) e 2 (27\%), lordose pré-revisão: $36^{\circ}$ média $44^{\circ}$ e pós-revisão. Pré-revisão: IP55, VP26 $6^{\circ}$ PS $29^{\circ}$ e pós-operatória IP56 $6^{\circ}$, VP22 e PS34 . Desequilíbrio sagital pré-revisão $60 \%$ e pós-operatório $27 \%$, equilíbrio, coronal recuperado nos 2 casos. A RM antes da primeira intervenção mostrou 83\% com Pfirmann IV. Nível da SA: 38\% L3-L4, L5-S1: 26\%. Tipos de SA: estenose lombar agravada pela ântero-listese monossegmentar em 20 casos e por retro-listese em 14, estenose multissegmentar 4, hérnias de disco 4, fraturas por esmagamento 4, estenoses sem listese 4. Período entre as 2 cirurgias: em média 6 anos. Conclusão: A incidência de 9,4\% de re-operação em cirurgias decorrentes de síndrome adjacente torna esta doença um problema importante a longo prazo, obrigando o cirurgião a tomar todas as medidas possíveis para evitar esta situação. Com os resultados deste estudo retrospectivo só poderíamos dizer que os segmentos mais propensos a desenvolver SA são os com Pfirmann IV na ressonância magnética e que se deve levar em conta a relação VP (20\%) e PS (80\%) pré e pós-operatória para voltar à condição mecânica perfeita da coluna vertebral. Estudos clínicos prospectivos são necessários para que se obtenham resultados mais conclusivos.

Descritores: Síndrome; Doenças da coluna vertebral, Cirurgia; Radiografia.

\begin{abstract}
Objective: We studied the incidence of lombar AS surgically treated and the factors associated with it. Method: Retrospective cross sectional study. Results: We found 50 surgeries for AS performed in 1662 cases, 530 in the lumbar spine, incidence: 9.4\%. Average age: 62 years. Main symptoms: radicular claudication (80\%). Radiographs prior to the first surgery level that will suffer AS: UCLA 1 (45\%) and 2 (27\%), lordosis pre-revision: $36^{\circ}$ average and $44^{\circ}$ post-revision. Pre-revision: IP $55^{\circ}$, VP $26^{\circ}$ and PS $29^{\circ}$ and postoperative IP $56^{\circ}$, VP $22^{\circ}$ and PS $34^{\circ}$. Sagittal imbalance pre-revision $60 \%$ and postoperative $27 \%$, coronal balance recovered in both cases. MRI before the first intervention was 83\% with Pfirmann IV. AS level: 38\% L3-L4, L5-S1: 26\%. AS types: lumbar stenosis worsened by monosegmental antero-listhesis in 20 and retro-listhesis in 14; multisegmental stenosis 4, disc herniation 4, crush fracture 4, stenosis without listhesis 4. Period between the 2 surgeries: on average 6 years. Conclusion: An incidence of $9.4 \%$ of reoperation due to adjacent syndrome makes this disease an important long-term problem, forcing the surgeon to take all the possible steps to avoid this. With the results of this retrospective study we could only say that the segments more likely to develop AS are those with Pfirmann IV on MRI and that the relation VP (20\%) and PS (80\%) pre and post-operative should be taken into account to return to the spinal column perfect mechanical condition. Prospective clinical studies are needed for more conclusive results.
\end{abstract}

Keywords: Syndrome; Spinal diseases, Surgery; Radiography.

1. Ex-felow-interno de la Unidad de Patología Raquidea del Hospital Pellegrin - Bordeaux, Francia; Centro de Patología Raquídea; Unidad de Patología Espinal - Hospital Padilla - Tucumán, Argentina. 2. Profesor del Hôpital Pellegrin, Unite de Pathologie Rachidienne, CHU - Bordeaux, Francia. 


\section{INTRODUCCION}

El síndrome adyacente (SDA) es el conjunto de síntomas y signos consecuentes a la degeneración del nivel contiguo a una cirugía espinal; tal vez sea la complicación a largo plazo mas frecuente en la cirugía de artrodesis lumbar. Teniendo en cuenta el aumento de las expectativas de vida, la mejor calidad de vida a edades mas avanzadas y los adelantos técnicos tanto quirúrgicos como anestésicos, ha llevado a que se amplíen en forma considerable los limites de edad en los que se puede someter a una cirugía de revisión al paciente portador de un SDA.

Si bien la degeneración del nivel adyacente en su gran mayoría es solo imagenológica (con prevalencia de hasta un 90\% según algunos autores) $)^{1-3}$ (Figura 1), el porcentaje de pacientes que presentan clínica es importante (puede alcanzar el 30\% a los 5 años) ${ }^{4}$ y llegado el momento de su solución quirúrgica se planteará como un problema complejo ante el cirujano. La complejidad proviene no solo del hecho que es una reintervención sino también de la obligación de identificar la causa del síndrome adyacente para intentar corregirla y/o no repetirla; aunque esto no siempre ocurra ya que la etiología del SDA es multifactorial y aún no claramente conocida ${ }^{1,2,4-6}$.

Si bien se podría adjudicar algunos factores predisponentes inherentes al paciente $e^{7-9}$, la mayor parte de la bibliografía muestra pruebas sobre el principal origen del SDA en las alteraciones mecánicas producidas luego de una cirugía que causan el deterioro del nivel móvil adyacente a la zona operada $a^{1,2,5,10-18}$.

El objetivo de este trabajo es estudiar la incidencia del SDA quirúrgico de localización lumbar en el Servicio de Cirugía Raquídea del Hospital Pellegrin de Bordeaux y los factores relacionados con este síndrome.

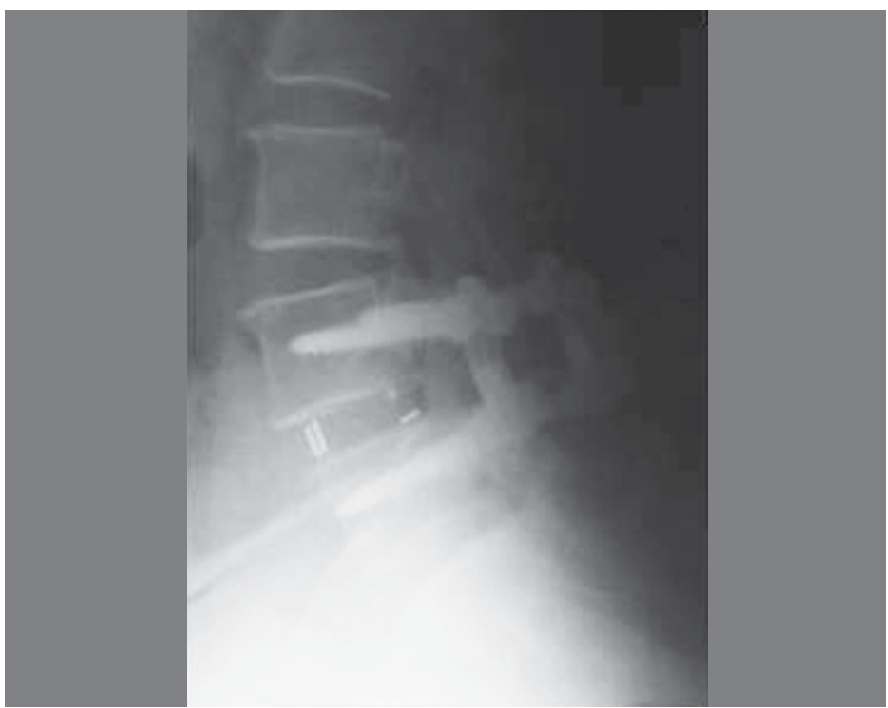

Figura 1. Syndrome adyacente en el segmento inmediatamente superior a la artodesis.

\section{MATERIAL Y METODOS}

Se realizó un estudio retrospectivo de corte transversal tomando todos los pacientes operados por síndrome adyacente de localización lumbar en el periodo de mayo 2008 a mayo 2009 en el servicio de cirugía raquídea del Hospital Pellegrin, $\mathrm{CHU}$ Bordeaux.

Para la determinación de la incidencia se reviso el programa quirúrgico del servicio durante el periodo de un año y luego se obtuvieron del archivo del hospital las historias clínicas de los pacientes identificados.

Se entendió como síndrome adyacente a toda degradación aguda o crónica de un nivel subyacente o suprayacente a una fusión (sea buscada o no), se tomaron solo aquellos casos cuyos limites (sea superior o inferior) estuviesen en la región lumbar.
Dentro de los diagnósticos de los diferentes tipos de síndromes adyacentes se consideraron:

- Espodilolistesis (antero, retro o latero);

- Estenosis degenerativa del canal lumbar;

- Fractura aplastamiento;

- Hernia discal;

- Pérdida del equilibrio sagital o coronal ${ }^{1,2,4,11,19}$.

Se estudiaron los antecedentes personales y familiares del paciente.

Para evaluar las radiografías preoperatorias (esto quiere decir: postoperatorias inmediatas a la primera cirugía de artrodesis) se clasificó el estado de degeneración de los espacios intervertebrales adyacentes mediante la escala UCL ${ }^{10}$ (Tabla 1) y se midió los ángulos de lordosis segmentaria y lumbar en placas en bipedestación al igual que la presencia o no de espondilolistesis y en caso de existencia de anterolistesis se la clasificó según el grado de Meyerding; las medidas del equilibrio sagital, coronal, incidencia pélvica, versión pélvica e inclinación sacra se tomaron directamente de los ángulos marcados por los técnicos especialistas en las placas EOS (aparato que permite la toma de radiografía de cuerpo entero en bipedestación con baja radiación). Se consideró desequilibrio sagital cuando la línea, conducto auditivos- cabeza femorales, estaba desplazada $3 \mathrm{~cm}$ hacia adelante o hacia atrás con las rodillas extendidas (esta línea se traza en forma sagital desde el punto medio de la línea de unión entre ambos conductos auditivos externos; luego se mide la distancia a que quedó el punto medio de la línea de unión del centro de ambas cabezas femorales con respecto a la sagital ya trazada) y mientras que el desequilibrio coronal se considero existente cuando la línea trazada desde los conductos auditivos externos pasaba a mas de $4 \mathrm{~cm}$ hacia izquierda o derecha de la línea media sacra ${ }^{20-23}$ (Figura 2)

En lo que respecta a el ángulo facetario se midió tomando la línea que pasa por los extremos anterior y posterior de la articulación superior de la vertebra y la línea media sagital que pasa por el centro del disco y sigue la apófisis espinosa (en caso de rotaciones vertebrales se tomo la sagital del cuerpo vertebral ) mediante el logiciel DxWin sobre los cortes axiales tomográficos (Figura 3) ${ }^{24}$

Para evaluar el estado del disco se utilizó la clasificación de pfirmann ${ }^{25}$ en las IRM de la primera cirugía, al igual que se observó la presencia o no de signos de modic en los platillos vertebrales ${ }^{26}$.

Se estudió también sobre qué tipo de cirugía se desarrollo el SDA y cuanto tiempo pasó entre la primera y la segunda intervención quirúrgica, así como también si hubo complicaciones en las mismas.

Todos estos datos de las variables estudiadas se volcaron en fichas individuales (Figura 4).

El grado de degeneración del espacio discal (estudiado en el par radiográfico) es asignado por el hallazgo radiográfico más severo.

Tabla 1. Escala de la Universidad Central de Los Ángeles (UCLA) para medir la degeneración del espacio intervertebral en las placas radiográficas.

\begin{tabular}{c|c|c|c}
\hline Grado & Disminución del espacio discal & Osteofitos & Esclerosis del patillo vertebral \\
\hline I & - & - & - \\
\hline$\|$ & + & - & - \\
\hline$I I$ & +- & + & - \\
\hline IV & +- & +- & +
\end{tabular}

El grado de degeneración del espacio discal (estudiado en el par radiográfico) es asignado por el hallazgo radiográfico más severo.

\section{RESULTADOS}

Durante el periodo de mayo 2007 a mayo 2008 se hicieron en el servicio 1662 cirugías de las cuales 50 tenían el diagnostico de síndrome adyacente (SDA) lumbar, escritas en el parte de quirófano como revisión de artrodesis de columna, prolongación de artrodesis de columna o simplemente síndrome adyacente, así podemos hablar de una incidencia del 3\% dentro de todas las patologías que se tratan quirúrgicamente en la unidad de patología raquídea.

Sabiendo que en promedio en el servicio se realizan por semana 

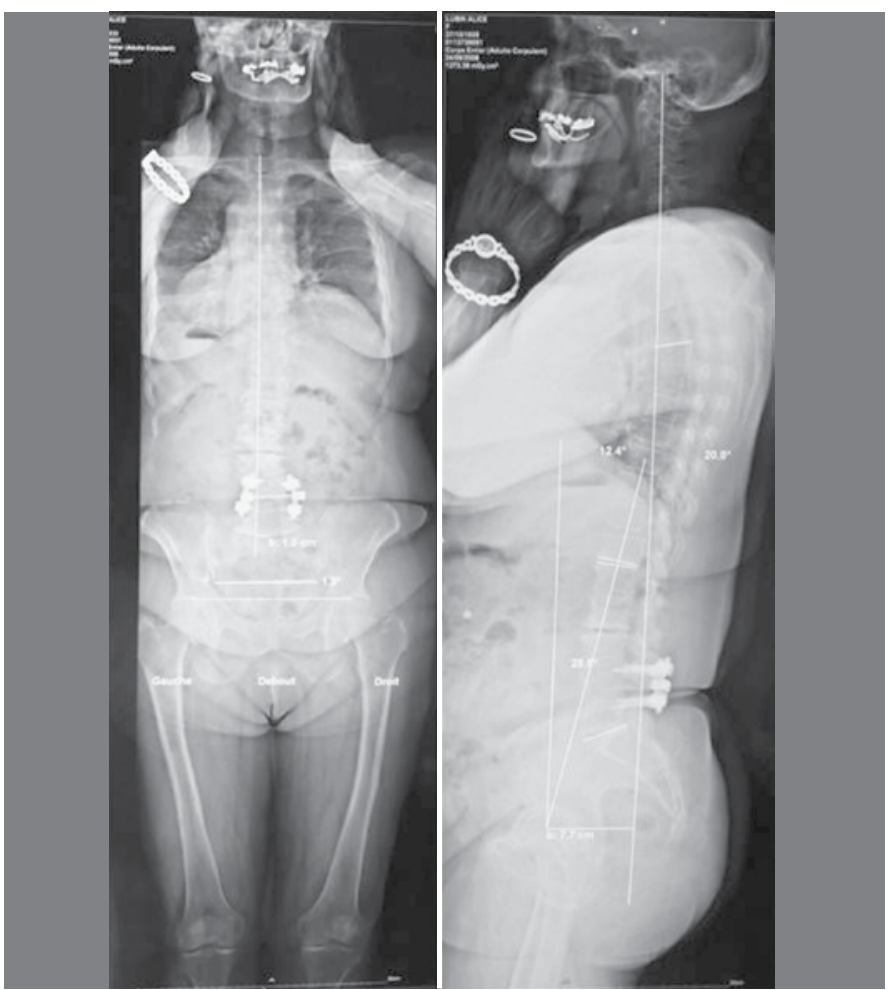

Figura 2. Placa EOS con medición del equilibrio sagital, coronal y lordosis.

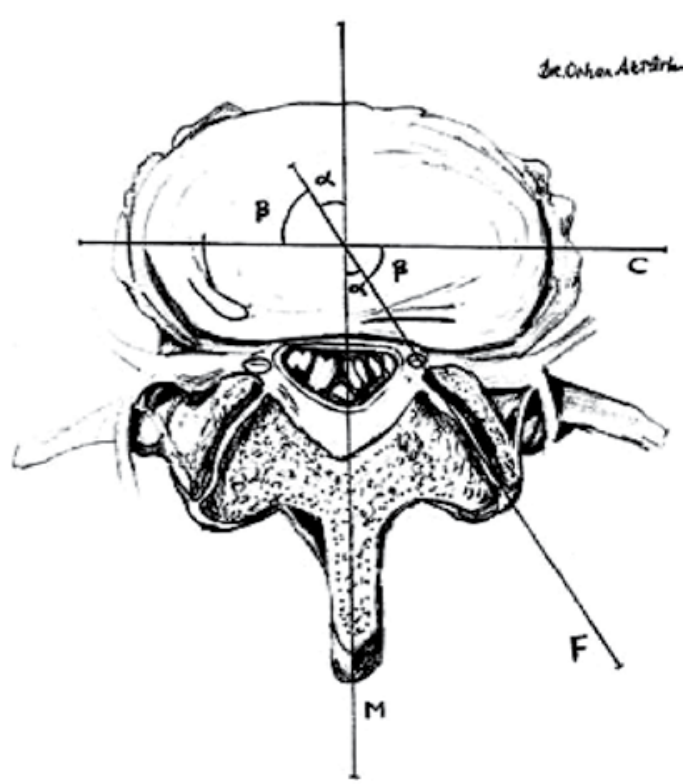

Figura 3. Método de medición del ángulo facetario representado por alfa donde la línea sagital es la que corta el centro del disco y espinosa mientras la línea que pasa por la articular debe tocar el punto anterior y posterior de la faceta superior de la vértebra inferior.

10 cirugías de artrodesis en las cuales algunos de sus extremos (proximal o distal) terminan en la región lumbar, podemos hacer el calculo de que un 9,4\% de las artrodesis lumbares serán reintervenidas quirúrgicamente a causa de un síndrome adyacente (haciendo la aclaración que el número de cirugías hace seis años era similar al actual).

Los pacientes estudiados fueron 37 mujeres (74\%) y 13 hombres (26\%) con edad promedio de 62 años (entre 14 a 87). El promedio de edad de las mujeres fue de 62 años y el de varones también.

Con respecto a los antecedentes y condiciones personales de

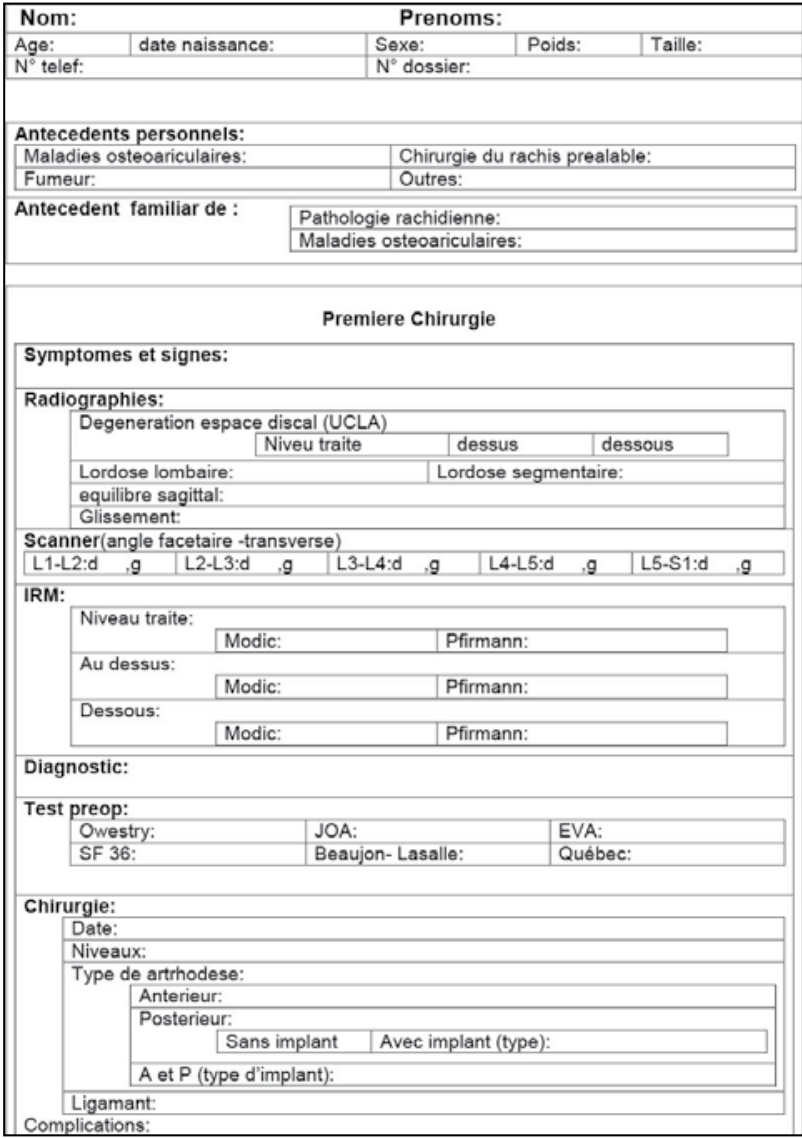

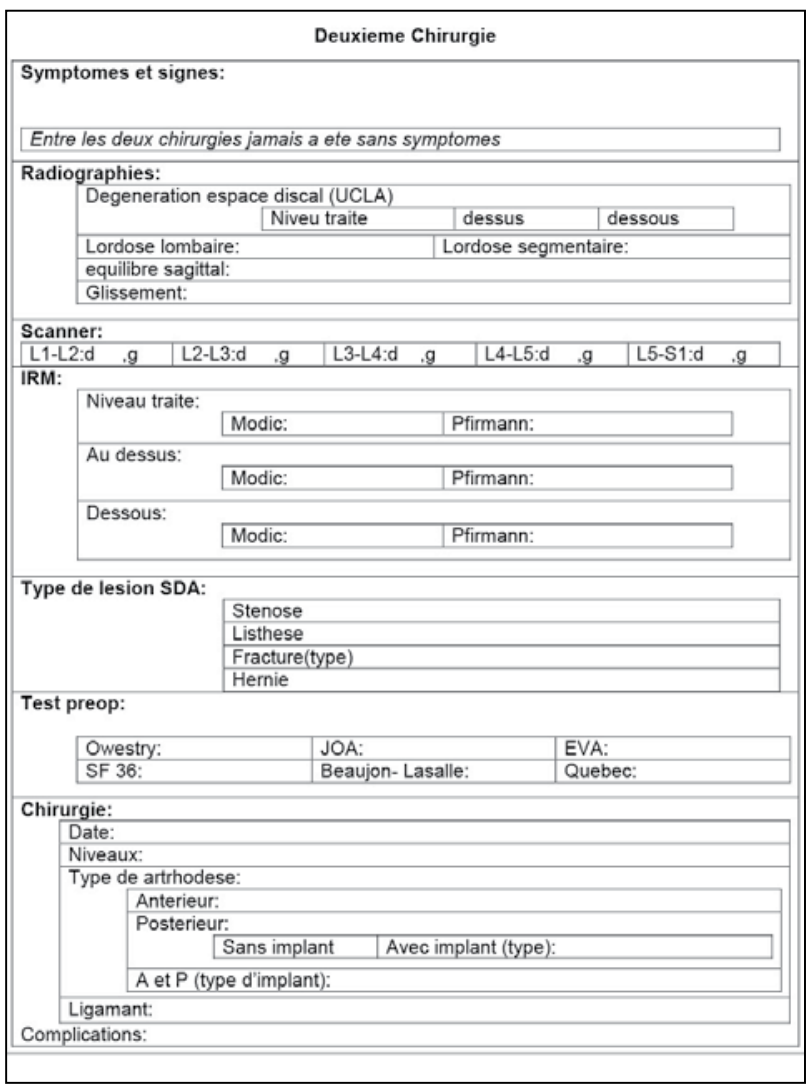

Figura 4. Ficha utilizada para cada paciente, con los datos extraídos de su historia clínica y de la medición de los estudios por imágenes. 
pacientes se encontró, 3 que habían sido operados por canal cervical estrecho, 5 fueron operados por hernia discal sin artrodesis, 6 pacientes presentaron una anomalía de charnela, 3 con diagnostico de depresión y 10 fumadores.

En lo que refiere a la primera artrodesis sobre la cual se desarrollo el síndrome adyacente, encontramos:

- Artrodesis posterior mas instrumentación con tornillos y barras (30 pacientes):L1L2: 1caso; L2L3L4: 1; L2-L5: 1; L3L4: 1; L3S1: 2; L4L5: 12; L4L5S1: 6; L3L4L5: 2; L5-S1: 3; T12-L5: 1; T10-L5: 1 ;

- Artrodesis posterior mas cuadro de Dove: L3L4L5, 2 casos;

- Artrodesis posterior con instrumentación de tornillos y barras mas caja TLIF o PLIF (11 pacientes): L3L4L5: 3 casos, L4L5S1: 2 casos, L3-S1: 1; L5S1:1; L1-S1: 1; L3L4: 1; T11-L5: 1; T10-L4: 1;

- Artrodesis posterior sin instrumentación: L4L5 2

- Artrodesis anterior: L4L5: 1 caso;

- Prótesis de disco L5S1 1 caso;

- Fusión natural: 2 casos (L4L5 y L5S1).(Figura 5)

En lo que refiere al número de niveles artrodesados que tenían los pacientes que fueron estudiados lo vemos en la tabla siguiente (Tabla 2):

La sintomatología que presentaron los pacientes fue de radiculalgias de esfuerzo en el $80 \%$ de los casos con territorio monoradicular de L4 en 12 casos; monoradicular de L5 en 8 casos y de S1 en 12 casos; el dolor fue bilateral en 6 casos de cruralgias y en 10 de ciatalgias; mientras que presentaron solo lumbalgia invalidante en 10 casos.

Un 45\% (22 casos) de los pacientes no tuvieron periodo asintomático entre las dos cirugías, esto quiere decir que en mayor o menor medida continuaron con sintomatología similar a aquella que llevo a la primera cirugía o por lo menos una lumbalgia.

El examen de las radiografías previas a la cirugía de revisión mostró que el nivel a tratar en un 39\% de los casos era ucla 2; 36\% ucla $4 ; 17 \%$ ucla 3 y en un $8 \%$ ucla 1 (Figura 6).

En cuanto al estadio ucla del nivel que sufrió la degeneración adyacente medido en las placas radiográficas previas a la primera artrodesis, se encontró que $45 \%$ era ucla $1,27 \%$ ucla $2,18 \%$ ucla 3 y $9 \%$ ucla 4 (Figura 7 ).

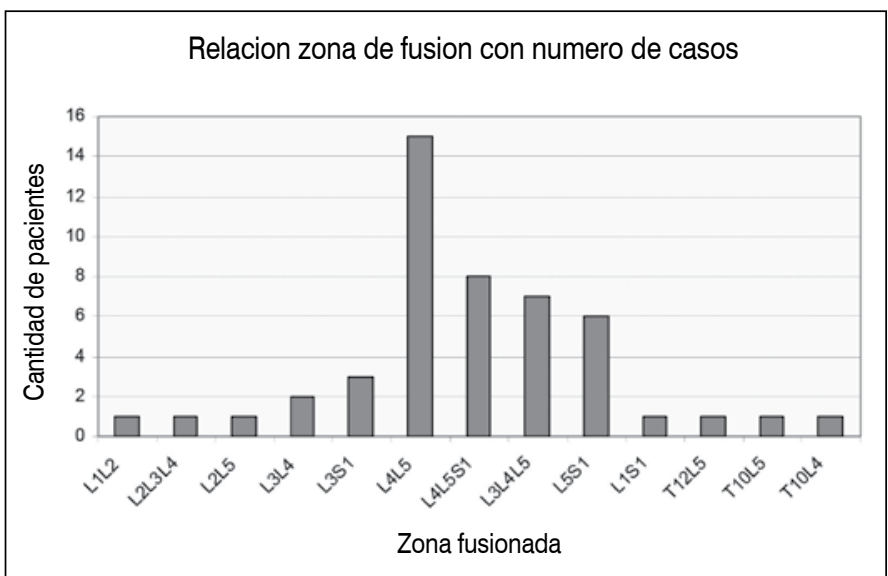

Figura 5. Es la relación entre la zona de la columna que fue fusionada sobre la cual se desarrolla el SDA y el numero de casos por zona.

Tabla 2. Cantidad de niveles artrodesados que presentaban los pacientes que desarrollaron un SDA.

\begin{tabular}{c|c}
\hline Numero de niveles artrodesados & Cantidad de casos \\
\hline Un nivel & 25 \\
\hline Dos niveles & 16 \\
\hline Tres o mas & 9 \\
\hline
\end{tabular}

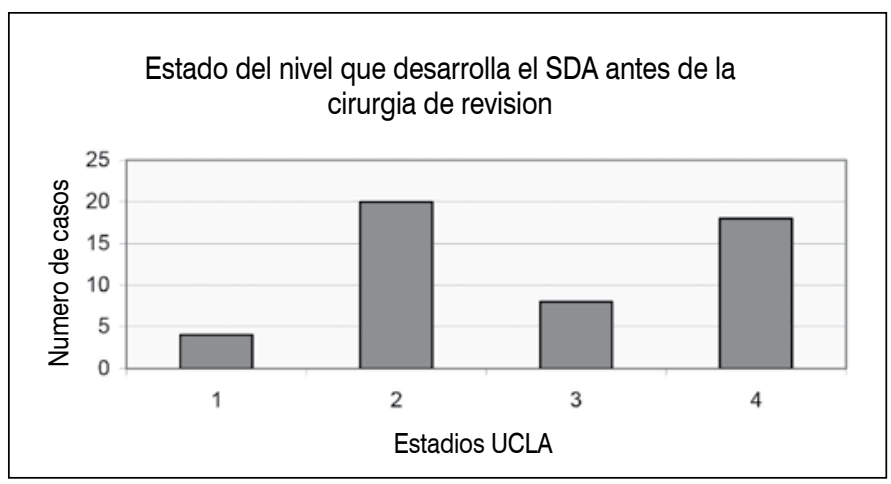

Figura 6. Relación entre el grado de degradación del nivel móvil y número de casos previo a cirugía de revisión.

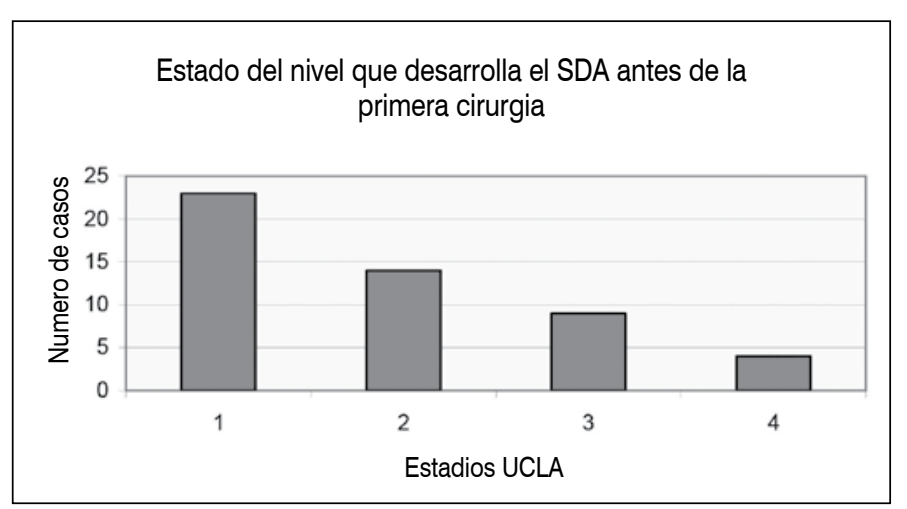

Figura 7. Relación entre degradación radiográfica del nivel móvil y el número de casos previo a primera cirugía.

La lordosis lumbar media previa a la cirugía de revisión fue de de $36^{\circ}$ (rango entre $80^{\circ}$ y $-31^{\circ}$ ) y luego de la cirugía fue de $44^{\circ}$ (con rangos entre $78^{\circ}$ y $10^{\circ}$ ), la lordosis del segmento a tratar fue en promedio previo a la cirugía de $28^{\circ}$ (rangos entre $127^{\circ}$ y $-32^{\circ}$ ) y luego de la cirugía $36^{\circ}$ (con rangos que van de 136 a $0^{\circ}$ ).

Al analizar las placas EOS se encontraron solo 20 casos preoperatorios y 11 controles postoperatorios en los cuales vimos que en el equilibrio sagital previo a la cirugía de revisión se había perdido en un $60 \%$ de los pacientes mientras que en el posquirúrgico solo un $27 \%$ seguía desequilibrado, la línea que va desde los conductos auditivos externos hasta las cabezas femorales en preoperatorio vario entre 25 a $-7,6 \mathrm{~cm}$ con un promedio de $2,2 \mathrm{~cm}$; en postoperatorio vario entre 3,9 a -7 con un promedio de $-1,6 \mathrm{~cm}$. Los valores de la incidencia pélvica (IP), versión pélvica (VP) e inclinación o pendiente sacra (PS) fueron:

- IP preop media $55^{\circ}$ con rangos de 76 a 31;

- VP preop media $26^{\circ}$ con rangos de 46 a 5;

- PS preop media $29^{\circ}$ con rangos de 51 a 7 ;

- IP postop media $56^{\circ}$ con rangos de 76 a 38;

- VP postop media $22^{\circ}$ con rangos de 42 a 3;

- $\quad$ PS postop $34^{\circ}$ con rangos de 57 a 20.

Cuando se analizo el equilibrio coronal en preoperatorio se vieron solo 2 casos con desequilibrio mientras que en postoperatorio ninguno.

Se encontró un 73\% (37) de listesis en el nivel de SDA, de las cuales $28 \%$ fueron retrolistesis ( $1 \mathrm{~L} 1 \mathrm{~L} 2$, $8 \mathrm{~L} 2 \mathrm{~L} 3,6 \mathrm{~L} 3 \mathrm{~L} 4)$ y un $45 \%$ antelistesis (1 L2L3, 11 L3L4, 5 L4L5, 5 L5S1) (Figura 8).

En el análisis del ángulo facetario se obtuvieron los valores promedio y máximos y mínimos de cada nivel lumbar discriminando entre derecha e izquierda (Tabla 3).

Cuando se analizo los estudios de IRM sobre la totalidad de pacientes solo se encontró 12 resonancias previas a la primera intervención y en las cuales se pudo ver que los niveles que de- 


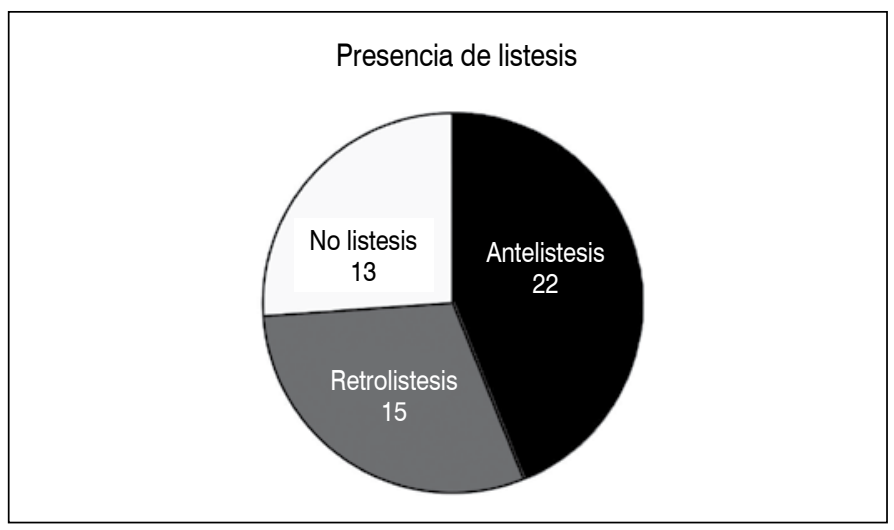

Figura 8. Presencia o no de listesis en el nivel con SDA.

Tabla 3. Angulo de inclinación de las facetas articulares con respecto a la sagital. Los valores promedios encontrados, discriminando el nivel y el lado.

\begin{tabular}{c|c|c|c|c|c|c|c|c|c|c}
\hline & \multicolumn{2}{|c|}{ L1L2 } & \multicolumn{2}{c|}{ L2L3 } & \multicolumn{2}{c|}{ L3L4 } & \multicolumn{2}{c|}{ L4L5 } & \multicolumn{2}{c}{ L5S1 } \\
\hline & Der. & Izq. & Der. & Izq. & Der. & Izq. & Der. & Izq. & Der. & Izq. \\
\hline promedio & $21^{\circ}$ & $25^{\circ}$ & $24^{\circ}$ & $30^{\circ}$ & $37^{\circ}$ & $35^{\circ}$ & $40^{\circ}$ & $41^{\circ}$ & $49^{\circ}$ & $52^{\circ}$ \\
\hline máximo & 32 & 43 & 41 & 46 & 56 & 52 & 66 & 70 & 67 & 76 \\
\hline mínimo & 11 & 10 & 13 & 12 & 11 & 13 & 18 & 10 & 18 & 20 \\
\hline
\end{tabular}

sarrollaron luego el SDA ya tenían un Pfirmann IV en 10 casos y un estadio $V$ en los otros dos casos, por el contrario ninguno tuvo signo de modic (Figura 9). Al analizar las IRM previas a la cirugía de revisión se encontro 20\% Modic I, 12\% Modic II y 68\% no presentaron Modic. El estadio de Pfirmann del disco en el nivel que sufrió el SDA fue 56\% IV, 28\% III y 16\% V (Figura 10).

Los niveles que sufrieron la degeneración adyacente se localizaron por arriba de la fusión en 27 pacientes, por abajo en 17, por arriba y también por abajo en 4 pacientes, finalmente solo en 2 pacientes se observo el SDA arriba pero intercalando un nivel.

En cuanto al nivel mas afectado por SDA fue L3L4, representando un 38\% con 19 casos seguido por L5S1 (Figura 11).

Los tipos de SDA que se presentaron fueron:

- Estenosis lumbar agravada por antelistesis en 20 casos;

- Estenosis lumbar agravada por retrolistesis en 14 casos:

- Estenosis lumbar a múltiples niveles en 4 casos (estenosis en 2 o mas niveles, incluye antelistesis en 2 casos y una retrolistesis);

- Hernia discal en 4 casos;

- Fractura aplastamiento en 4 casos;

- Estenosis lumbar a un nivel 4 casos (Figura 12).

El periodo transcurrido entre la primera cirugía y la cirugía de revisión fue en promedio 6 años con un máximo de 19 años y un mínimo de 2 meses.

El tratamiento quirúrgico de revisión fue en 19 casos (38\%) fijación del nivel comprometido mediante tornillos y barras con injerto posterolateral; en 14 (28\%) pacientes se fijaron 2 o mas niveles con tornillos, barras, cajas íntersomáticas (TLIF) e injerto posterolateral(en 4 de estos pacientes se realizaron osteotomías de corrección); en 7 casos se fijo el nivel con SDA en $360^{\circ}$ utilizando tornillos, barras, caja e injerto posterolateral; en 5 pacientes se fijaron 2 o mas niveles solo con tornillos, barras e injerto óseo, por ultimo en 5 pacientes se utilizaron sistemas híbridos de fijación + ligamento (DTO o BDYN) (Figura 13).

Las complicaciones que se presentaron luego de estas cirugías de SDA fueron 17 (34\%): en 3 ocasiones una fractura por aplastamiento por arriba de la zona artrodesada; segunda cirugía de revisión a causa de un nuevo SDA en 1 pacientes; reintervención por mala posición de un tornillo en 2 casos; hematoraquis en 1 oportunidad; infección en $3(6 \%)$ casos; degeneración sintomática del nivel adyacente en 2 casos y persistencia de la sintomatología que llevo a la cirugía en 4 casos.

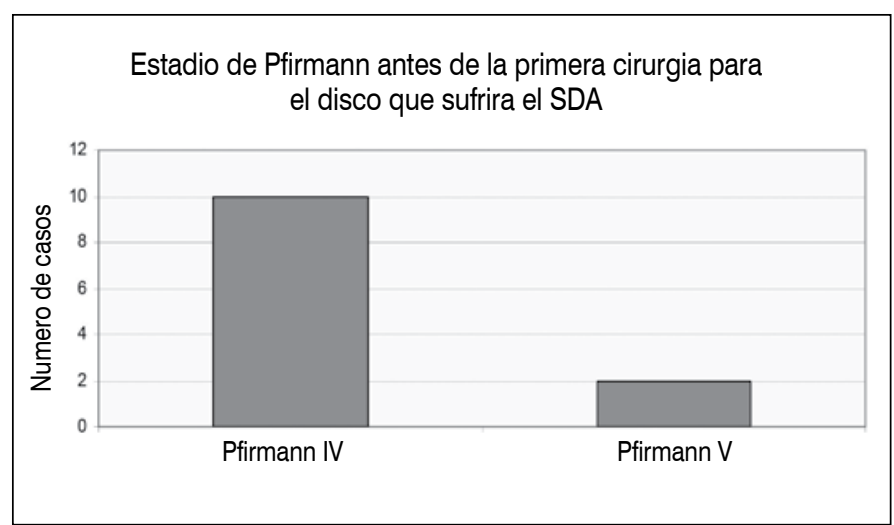

Figura 9. La degeneración discal del nivel que sufrirá el SDA antes de la primera cirugía de fijación, medida en la IRM a través de la clasificación de Pfirmann (n 12).

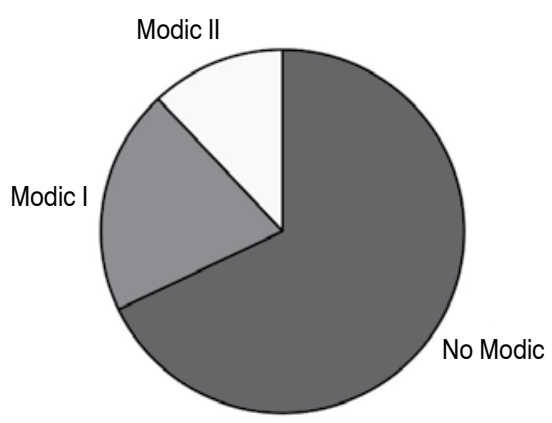

Figura 10. La presencia del signo de Modic en las IRM previas a la cirugía de revisión (n 50).

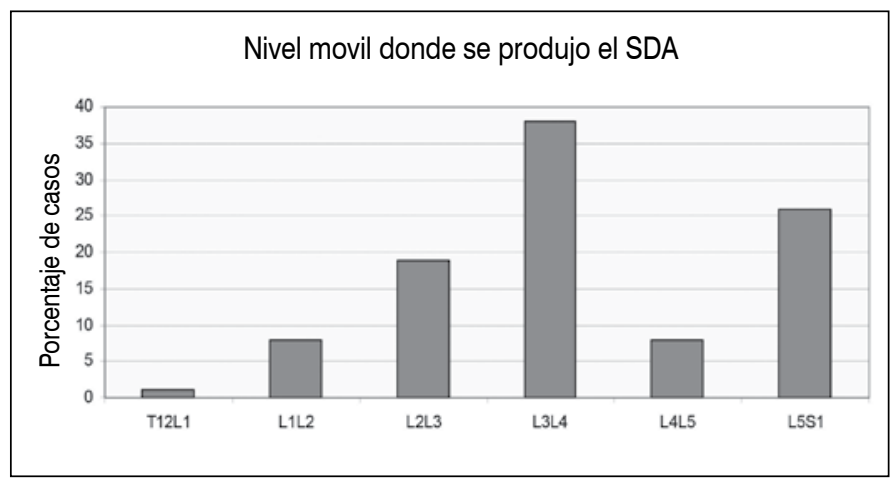

Figura 11. Niveles afectados por SDA.

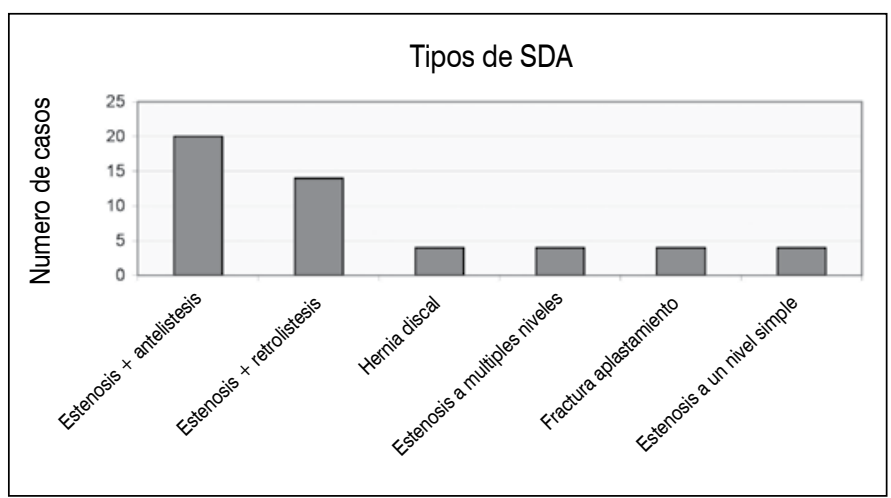

Figura 12. Los Tipos de SDA que se encontraron por número de casos. 


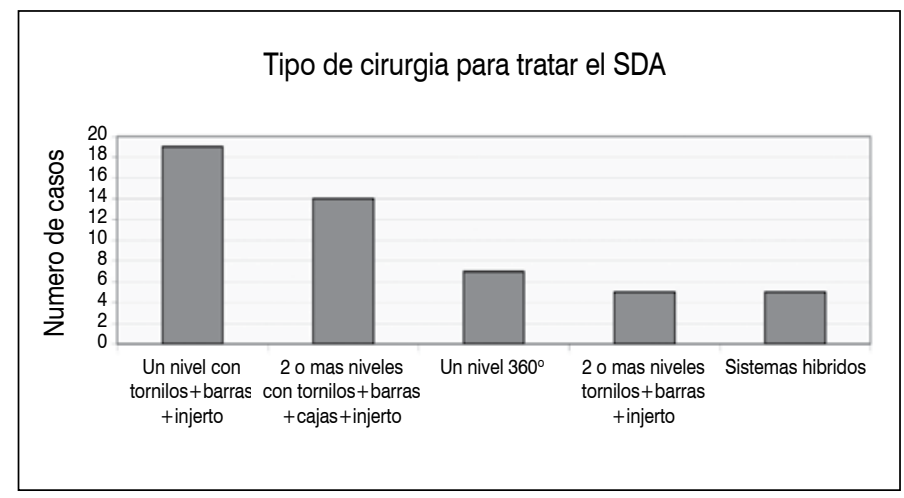

Figura 13. El tipo de cirugía realizada para el tratamiento del SDA.

\section{DISCUSION}

Cuando se habla del Síndrome adyacente se debe hacer bien la aclaración de su calidad de "síndrome" que por definición es un conjunto de síntomas y signos por lo que no cabría en la misma una degeneración radiológica del nivel adyacente a una fusión sin manifestaciones clínicas. Éste sintomatología al no responder al tratamiento médico puede terminar en una resolución quirúrgica. En la literatura se ha encontrado múltiples definiciones como "degeneración del segmento adyacente (ASD en la literatura angloparlante)" que podría ser solo radiológica sin ninguna importancia clínica o el término de "Deterioro del Segmento Adyacente" que puede englobar tanto pacientes sintomáticos como los no sintomáticos ${ }^{17,18}$. Otro nombre con el cual se lo identifico es como" patología de la zona de transición" lo que no nos deja una idea clara del concepto, tal vez uno de los términos mas acertados en la bibliografía sea el de "enfermedad del segmento adyacente"(ASDisease).

Con respecto a la incidencia de casos por año en el servicio se encontró 9,4\%, valor coincide con el de la literatura que habla de entre un 5 a $15 \%$ de revisiones por SDA ${ }^{1,2,5}$.

La edad promedio de 62 años nos habla del territorio degenerativo sobre el cual se desarrolla esta patología y no encontramos diferencia entre varones y mujeres por lo que no podemos hablar de la influencia postmenopáusica como Etebar e Cahill ${ }^{27}$

La zona de fusión más frecuente de la primera artrodesis esta perfectamente relacionada con la predominancia de patología degenerativa del adulto que se maneja en el servicio, al igual que su sintomatología.

Un dato no menor es el $45 \%$ de pacientes que no tuvieron un periodo libre de sintomatología entre sus 2 cirugías, lo que podría hablar sobre un pobre resultado de la primera intervención o una personalidad demandante o débil del paciente, cosas que llevarían a elevar las posibilidades de una segunda cirugía.

En las placas radiográficas previas a la primera cirugía y a la segunda, el estado del disco en su mayoría se mantuvo entre los estadios 1 y 2 de UCLA los cuales son discos móviles y con capacidad de deslizamientos y compresiones por ante o retrolistesis; el alto numero de UCLA 4 previos a la cirugía de revisión se explicaría quizás por ser niveles que ya hicieron su deslizamiento.

El valor promedio de lordosis fue bajo debido a la edad promedio y la evolución natural a la perdida de la misma con la artrosis sumada a las patologías propias de los pacientes como la antelistesis, sin embargo luego de las cirugías se eleva unos 8 grados el promedio, mostrando los esfuerzos en la corrección de la cirugía de revisión. Los valores de la lordosis segmentaria tal vez no fueron muy claros debido a que la lordosis entre L5 y SI se midió entre el platillo superior de L5 y el muro posterior del sacro, lo que da angulaciones completamente desfasadas con respecto al resto de los niveles.

El análisis del equilibrio sagital mediante las placas EOS mostró claramente como se logra con cierto éxito volver hacia atrás a la línea sagital en un 33\% de los pacientes con un promedio de -1,6 cm hacia atrás de las cabezas femorales, lo que seria perfectamente normal, y también se devuelve el equilibrio coronal en los dos casos que lo habían perdido. Analizando los promedios de incidencia pelviana vemos que los pacientes se ajustaron estrictamente a la normalidad en la dimensión de su pelvis, inclusive los valores con respecto a nuevas mediciones en los postoperatorios son idénticas con lo que se eliminaría la posibilidad de errores en las mediciones; el verdadero problema se plantea en la relación entre Versión pelviana y Pendiente sacra, ya que aquí los valores están alterados pues la relación normalmente debe ser $20 \% \mathrm{VP}+80 \% \mathrm{PS}=100 \%$ IP , esto esta totalmente perdido en las preoperatorias con $47 \%$ VP $+53 \%$ PS y en las postoperatoria esto se modifica ligeramente con $39 \% \mathrm{VP}+61 \% \mathrm{PS}^{20,28}$. Las alteraciones de las proporciones serían producto de la puesta en marcha de los mecanismos de compensación y el paciente logra mantener la línea sagital a una distancia normal de las cabezas femorales ${ }^{15}$. Ahora podríamos preguntarnos si realmente esto es un equilibrio bien compensado o si es un "desequilibrio encubierto" ya que se podría decir que los recursos de compensación de la columna se han agotado y no hay retorno desde este punto, las caderas y rodillas ayudarían de forma poco eficaz para devolver una línea sagital centrada pero solo seria un "camuflaje" de "normalidad". Suponiendo que esto fuera así , llegado el momento de la planificación quirúrgica de un paciente habría que prestar especial atención a devolverle las proporciones correctas de VP $20 \%+$ PS $80 \%=I P$ con las técnicas quirúrgicas necesarias para el caso (osteotomías, cajas, etc.) ${ }^{21,22}$. Tal vez una utilidad practica seria en saber que los niveles que son dudosos "borderline" deberían ser tomados en la primera cirugía si las condiciones de las proporciones VP+PS están alteradas (aún cuando la línea sagital sea correcta y la lordosis sea de valores normales) ya que podemos pensar que el deterioro en el equilibrio va a continuar irreversiblemente y las fuerzas de cizallamiento sobre el nivel móvil van a ser cada vez mayores, lo que llevara a un SDA de manera precoz ${ }^{19}$.

La presencia de listesis en un 73\% de los SDA habla de su carácter degenerativo, o en algún caso también podría hablar de inestabilidad iatrogénica después de la primera cirugía ${ }^{29}$

Con respecto a los ángulos de inclinación facetaria, encontramos que su medición es difícil y mas aun cuando se tratan de articulaciones artrósicas, sin embargo hemos obtenido valores promedios relativamente coherentes y dentro de la normalidad ${ }^{24}$; por otra parte hemos visto que en las escoliosis hay una gran diferencia entre el ángulo de la articular derecha y la articular izquierda del mismo nivel, es que es causa o efecto de la escoliosis degenerativa?, pero eso sería un tema a desarrollar en otro trabajo.

Con respecto a las IRM previas a la primera cirugía, que serian las de mayor valor predictivo, vimos que parece ser el estadio Pfirmann IV el más peligroso para luego desarrollar un SDA a posteriori, lo que tiene su lógica explicación en que ya es un disco dañado pero con una altura suficiente como para luego deslizarse con la listesis y producir la compresión. El signo de Modic no mostró ninguna relación importante.

Los niveles móviles (L3L4 y L5S1) donde más se produjeron los SDA corresponden coherentemente al nivel que fue más veces fijado aunque con un predominio de el nivel adyacente superior a la artrodesis, lo cual se podría explicar por la menor resistencia mecánica de los niveles superiores.

Un dato aislado que quizás sea casual pero debería estudiarse mas en profundidad es el hecho que no hubo artrodesis L4L5 en $360^{\circ}$ con SDA.

Con todos los datos anteriores es comprensible que el tipo de SDA que más se encontró fue una estenosis lumbar agravada por listesis, mientras que las fracturas aplastamientos se dieron en las cercanías de la charnela toracolumbar donde hay una mayor rigidez.

El período promedio transcurrido entre las dos cirugías esta dentro de los citados por la bibliografía consultada.

En los tipos de cirugías realizadas para el tratamiento quirúrgico del SDA se puede ver un porcentaje importante de cirugías de 20 mas niveles con utilización de cajas con o sin osteotomías lo que 
habla del alto grado de complejidad de la cirugía de SDA y del intento de corrección de un equilibrio alterado.

Por último las complicaciones de la cirugía de revisión son aún muy tempranas pero es de destacar el alto porcentaje de infección (6\%) y las 3 fracturas por aplastamiento que se localizaron todas ellas en cercanías de la charnela toracolumbar.

\section{CONCLUSIÓN}

Una incidencia de 9,4\% de reintervenciones en cirugías lumba- res a causa de un Sindrome Adyacente hace de esta patología un problema a largo plazo importante, lo que obligaría al cirujano a tomar las medidas que sean posibles para evitarlo.

Con los resultados de este estudio retrospectivo solo podríamos decir que tendrían más posibilidades de desarrollar un SDA los segmentos con Pfirmann IV en la IRM y que se debería tener en cuenta la relación VP (20\%) y PS (80\%) pre y post operatorias para devolver las condiciones mecánicas ideales al raquis.

Se necesitarían estudios clínicos prospectivos para conclusiones mas contundentes.

\section{REFERENCIAS}

1. Park P, Garton HJ, Gala VC, Hoff JT, McGillicuddy JE. Adjacent segment disease after lumbar or lumbosacral fusion: review of the literature. Spine (Phila Pa 1976). 2004 1:29(17):1938-44.

2. Guigui $P$, Lambert $P$, Lassale $B$, Deburge A. [Long-term outcome at adjacent levels of lumbar arthrodesis]. Rev Chir Orthop Reparatrice Appar Mot. 1997;83(8):685-96.

3. Miyakoshi N, Abe E, Shimada Y, Okuyama K, Suzuki T, Sato K. Outcome of one-level posterior lumbar interbody fusion for spondylolisthesis and postoperative intervertebral disc degeneration adjacent to the fusion. Spine (Phila Pa 1976). 2000 15;25(14):1837-42.

4. Cheh G, Bridwell KH, Lenke LG, Buchowski JM, Daubs MD, Kim Y,et al. Adjacent segment disease followinglumbar/thoracolumbar fusion with pedicle screw instrumentation: a minimum 5-year follow-up. Spine (Phila Pa 1976). 2007;32(20):2253-7.

5. Guigui $P$, Wodecki $P$, Bizot $P$, Lambert $P$, Chaumeil G, Deburge A. [Long-term influence of associated arthrodesis on adjacent segments in the treatment of lumbar stenosis: a series of 127 cases with 9-year follow-up]. Rev Chir Orthop Reparatrice Appar Mot. 2000;86(6):546-57.

6. Gille O, Jolivet E, Dousset V, Degrise C, Obeid I, Vital JM,et al. Erector spinae muscle changes on magnetic resonance imaging following lumbar surgery through a posterior approach. Spine (Phila Pa 1976). 2007;32(11):1236-41.

7. Penta M, Sandhu A, Fraser RD. Magnetic resonance imaging assessment of disc degeneration 10 years after anterior lumbar interbody fusion. Spine (Phila Pa 1976). 1995;20(6):743-7.

8. Van Horn JR, Bohnen LM. The development of discopathy in lumbar discs adjacent to a lumbar anterior interbody spondylodesis. A retrospective matched-pair study with a postoperative follow-up of 16 years. Acta Orthop Belg. 1992;58(3):280-6.

9. Boden SD, Davis DO, Dina TS, Patronas NJ, Wiesel SW. Abnormal magnetic-resonance scans of the lumbar spine in asymptomatic subjects. A prospective investigation. J Bone Joint Surg Am. 1990;72(3):403-8.

10. Ghiselli G, Wang JC, Bhatia NN, Hsu WK, Dawson EG. Adjacent segment degeneration in the lumbar spine. J Bone Joint Surg Am. 2004;86(7):1497-503.

11. Ruberté LM, Natarajan RN, Andersson GB. Influence of single-level lumbar degenerative disc disease on the behavior of the adjacent segments--a finite element model study. $J$ Biomech. 2009 9:42(3):341-8.

12. Aota $Y$, Kumano $K$, Hirabayashi S. Postfusion instability at the adjacent segments after rigid pedicle screw fixation for degenerative lumbar spinal disorders. J Spinal Disord. 1995:8(6):464-73.

13. Frymoyer JW, Hanley E, Howe J, Kuhlmann D, Matteri R. Disc excision and spine fusion in the management of lumbar disc disease. A minimum ten-year followup. Spine (Phila Pa 1976) 1978:3(1):1-6.

14. Hambly MF, Wiltse LL, Raghavan N, Schneiderman G, Koenig C. The transition zone above a lumbosacral fusion. Spine (Phila Pa 1976). 1998 15;23(16):1785-92.
15. Legaye J, Duval-Beaupère G, Hecquet J, Marty C. Pelvic incidence: a fundamental pelvic parameter for three-dimensional regulation of spinal sagittal curves. Eur Spine J. 1998;7(2):99-103.

16. Cunningham BW, Kotani Y, McNulty PS, Cappuccino A, McAfee PC. The effect of spinal destabilization and instrumentation on lumbar intradiscal pressure: an in vitro biomechanical analysis. Spine (Phila Pa 1976). 1997;22(22):2655-63.

17. Phillips FM, Reuben J, Wetzel FT. Intervertebral disc degeneration adjacent to a lumbar fusion. An experimental rabbit model. J Bone Joint Surg Br. 2002;84(2):289-94.

18. Marcovschi Champain S. Correlations entre les parameters biomecaniques du rachis et les indices cliniques pour l'analyse quantitative des pathologies du rachis lombaire et de leur traitement chirurgical [thèse].Paris: Laboratoire de Bio-Mécanique, ENSAM CER de Paris, CNRS; 2008

19. Kumar MN, Baklanov A, Chopin D. Correlation between sagittal plane changes and adjacent segment degeneration following lumbar spine fusion. Eur Spine J. 2001;10(4):314-9.

20. Gangnet N, Pomero V, Dumas R, SkalliW, Vital JM. Variability of the spine and pelvis location with respect to the gravity line: a three-dimensional stereoradiographic study using a force platform. Surg Radiol Anat. 2003;25(5-6):424-33.

21. Vital JM, Gille O, Gangnet N. Equilibre Sagittal et applications cliniques. Rev Rhumatisme. 2004:71:120-8

22. Gangnet N, Dumas R, Pomero V, Mitulescu A, Skalli W, Vital JM. Three-dimensional spinal and pelvic alignment in an asymptomatic population. Spine (Phila Pa 1976). 2006 1:31(15):E507-12

23. Roussouly P, Gollogly S, Berthonnaud E, Dimnet J. Classification of the normal variation in the sagittal alignment of the human lumbar spine and pelvis in the standing position. Spine (Phila Pa 1976). 2005 1;30(3):346-53

24. Karacan I, Aydin T, Sahin Z, Cidem M, Koyuncu H, Aktas I, et al. Uludag M. Facet angles in lumbar disc herniation: their relation to anthropometric features. Spine (Phila Pa 1976). 2004:29(10):1132-6.

25. Pfirrmann CW, Metzdorf A, Zanetti M, Hodler J, Boos N. Magnetic resonance classification of lumbar intervertebral disc degeneration. Spine (Phila Pa 1976). 2001;26(17):1873-8.

26. Modic MT, Steinberg PM, Ross JS, MasarykTJ, Carter JR. Degenerative disk disease: assessment of changes in vertebral body marrow with MR imaging.Radiology. 1988;166(1 Pt 1):193-9.

27. Etebar S, Cahill DW. Risk factors for adjacent-segment failure following lumbar fixation with rigid instrumentation for degenerative instability. J Neurosurg. 1999;90(Suppl 2):163-9.

28. Skalli W,Champain S, Mosnier T. Biomecanique du rachis. Paris: Du pathologie rachidienne; 2007.

29. Shah RR, Mohammed S, Saifuddin A, Taylor BA. Radiologic evaluation of adjacent superior segment facet joint violation following transpedicular instrumentation of the lumbar spine. Spine (Phila Pa 1976). 2003 1;28(3):272-5 